Pacific Journal of Mathematics

A TOPOLOGICAL BOUND ON THE NUMBER OF DISTINCT
ZEROS OF AN ANALYTIC FUNCTION 


\title{
A TOPOLOGICAL BOUND ON THE NUMBER OF DISTINCT ZEROS OF AN ANALYTIC FUNCTION
}

\author{
ROBERT F. BROWN
}

\begin{abstract}
An old theorem concerning the number of fixed points of a map on an annulus is used to obtain a lower bound for the number of distinct zeros of an analytic function. When the function is a polynomial, the result furnishes sufficient conditions on the coefficients so that the polynomial has at least a specific number of zeros.
\end{abstract}

Let $\mathbf{C}$ denote the complex numbers and, for real numbers $r, R$ with $0<r<R$, let

$$
A=A_{r, R}=\{z \in \mathbf{C}|r \leq| z \mid \leq R\} .
$$

It has long been known [2] that a (continuous) map $F: A \rightarrow A$ has at least $|\operatorname{deg}(F)-1|$ fixed points, where $\operatorname{deg}(F)$ denotes the degree of $F$ (see [3; page 34] for a modern proof).

We will apply this theorem to complex functions in order to obtain information about the number of zeros. There are well-known results, such as Rouché's Theorem [1], which count the number of zeros of complex functions. These results, however, count each zero as many times as its multiplicity. In contrast, the information we obtain is always in terms of distinct zeros.

I thank Alfred Hales for helpful discussions concerning this material.

Let $\Omega$ be a region (open, connected subset of $\mathbf{C}$ ) containing the origin. Suppose $f(z)$ is a complex function analytic on $\Omega$. By Taylor's Theorem, for each positive integer $k$ there is a polynomial $P(z)$, of degree less than or equal to $k$ (a Taylor polynomial of $f(z)$ at the origin), such that

$$
f(z)=P(z)+z^{k+1} g(z)
$$

for all $z$ in $\Omega$, where $g(z)$ is analytic on $\Omega$. We will refer to $(*)$ as a Taylor decomposition of $f(z)$. The function $Q(z)$ defined by

$$
Q(z)=-z^{-k} P(z) / g(z)
$$

will be called a Taylor quotient of $f(z)$. A fixed point of $Q(z)$ is a zero of $f(z)$. 
Proposition 1. Let $f(z)$ be analytic on a region $\Omega$ containing the origin. Suppose there exist $0<r<R$ and a Taylor decomposition $f(z)=$ $P(z)+z^{k+1} g(z)$ such that

(0) $|z| \leq R$ implies $z \in \Omega$

(1) $r \leq|z| \leq R$ implies $P(z) \neq 0$ and $g(z) \neq 0$

(2) $|z|=R$ implies $P(z)+\lambda z^{k+1} g(z) \neq 0$ for all $\lambda>1$

(3) $|z|=r$ implies $P(z)+\mu z^{k+1} g(z) \neq 0$ for all $0<\mu<1$ then $f(z)$ has at least $|\operatorname{deg}(Q)-1|$ distinct zeros $z$ with $r \leq|z| \leq R$, where $\operatorname{deg}(Q)$ denotes the degree of $Q(z)$ as a map of $A_{r, R}$ into $\mathbf{C}-0$.

Proof. The Taylor quotient of $f(z)$ is a well-defined map $Q: A_{r, R} \rightarrow$ $\mathbf{C}-0$ by hypotheses (0) and (1). Define $\rho: \mathbf{C}-0 \rightarrow A_{r, R}$ by

$$
\rho(z)= \begin{cases}\frac{r}{|z|} z & \text { if } 0<|z| \leq r \\ z & \text { if } r \leq|z| \leq R \\ \frac{R}{|z|} z & \text { if }|z| \geq R .\end{cases}
$$

Then, by [2], the map $\rho Q$ has at least $|\operatorname{deg}(\rho Q)-1|=|\operatorname{deg}(Q)-1|$ fixed points. Hypotheses (2) and (3) imply that a fixed point of $\rho Q$ is a fixed point of $Q$.

Proposition 1 seems difficult to work with. Even if we could verify those rather elusive hypotheses, we still have no guidance in computing the degree of the Taylor quotient $Q(z)$. Consequently, we will instead make use of the following rather weaker result.

Proposition 2. Let $f(z)$ be analytic on a region $\Omega$ containing the origin. Suppose there exists $R>0$ and a Taylor decomposition $f(z)=P(z)$ $+z^{k+1} g(z)$ such that

(0) $|z| \leq R$ implies $z \in \Omega$

(1) $|z| \leq R$ implies $P(z) \neq 0$ and $g(z) \neq 0$

(2) $|z|=R$ implies $|P(z)| \leq|g(z)| R^{k+1}$ then $f(z)$ has at least $k+1$ distinct zeros $z$ with $0<|z| \leq R$.

Proof. Since Hypotheses (0), (1) and (2) clearly imply the corresponding hypotheses of Proposition 1, in order to show that Proposition 2 is indeed a special case, it remains only to find $r$ satisfying $0<r<R$ for which (3) holds. Let $P(0)=a$ and $g(0)=b$, then $a$ and $b$ are nonzero by hypothesis and there exist positive real numbers $r_{1}$ and $r_{2}$, both smaller 
than $R$, such that

$$
\begin{array}{cc}
|z|<r_{1} \text { implies } & |P(z)|>|a / 2| \\
|z|<r_{2} & \text { implies }|g(z)|<|2 b| .
\end{array}
$$

Let $r$ be the smallest of $r_{1}, r_{2}$, and $|a / 4 b|^{1 / k+1}$, then

$$
|z|=r \text { implies }|P(z)|>|g(z)| r^{k+1}
$$

so (3) is certainly satisfied for this choice of $r$. The conclusion that $f(z)$ has at least $k+1$ zeros will now follow from Proposition 1 once we show that the degree of the Taylor quotient $Q(z)$ on $A_{r, R}$ is $-k$. To prove it, set $P^{+}(z)=P(z)-a$ and $g^{+}(z)=g(z)-b$. Choose $s>0$ so that $|z|<s$ implies both $\left|P^{+}(z)\right|<|a / 2|$ and $\left|g^{+}(z)\right|<|b / 2|$. Define $J_{s}=\{z \in \mathbf{C}|| z \mid$ $=s\}$ and consider the homotopy $H: J_{s} \times I \rightarrow \mathrm{C}-0$ given by

$$
H(z, t)=-z^{-k}\left(b+t g^{+}(z)\right)^{-1}\left(a+t P^{+}(z)\right) .
$$

Thus $Q$ on $A_{r, R}$, which is of the same degree as $Q$ restricted to $J_{s}$, is therefore of the same degree as the map $h: J_{s} \rightarrow \mathbf{C}-0$ defined by $h(z)=-(a / b) z^{-k}$. It is easy to see that $\operatorname{deg}(h)=-k$.

REMARKS. (1) If $P(0) \neq 0$ but $g(0)=0$, then $g(z)=z^{j} h(z)$ where $j$ is the multiplicity of 0 as a zero of $g(z)$. Thus we can write $f(z)=P(z)+$ $z^{k+j+1} h(z)$. Proposition 2 now applies to this Taylor decomposition so in fact $f(z)$ has at least $k+j+1$ distinct zeros $z$ with $0<|z| \leq R$.

(2) If $P(0)=0$, let $j$ be the multiplicity of 0 and write

$$
f(z)=z^{j}\left(S(z)+z^{k-j+1} g(z)\right)
$$

so $z^{j} S(z)=P(z)$. Consider $h(z)=S(z)+z^{k-j+1} g(z)$ which is a Taylor decomposition. Remark (1) assures us that we may assume $g(0) \neq 0$. Thus Proposition 2 applies to $h(z)$ and we can still conclude something about the zeros $z$ of $f(z)$ with $0<|z| \leq R$, namely, there are at least $k-j+1$ of them.

(3) It is easy to find examples of functions $f(z)$ satisfying the hypotheses of Proposition 2. We will next examine the situation when $f(z)$ is a polynomial. For a nonpolynomial example, let

$$
f(z)=2+z^{k}+3 e^{z+1} z^{k+1}
$$

then Proposition 2 implies that $f(z)$ has at least $k+1$ zeros $z$ such that $0<|z| \leq 1$.

A Taylor decomposition of a polynomial

$$
f(z)=a_{0}+a_{1} z+\cdots+a_{n} z^{n}
$$


is of the form

$$
\begin{aligned}
f(z)= & \left(a_{0}+a_{1}+\cdots+a_{k} z^{k}\right) \\
& +z^{k+1}\left(a_{k+1}+a_{k+2} z+\cdots+a_{n} z^{n-k-1}\right) .
\end{aligned}
$$

So, in this setting, Proposition 2 becomes

Proposition 3. Given $f(z)=a_{0}+a_{1} z+\cdots+a_{n} z^{n}$, suppose there exists $k$ with $0<k<n$ and $R>0$ such that

(1) $|z| \leq R$ implies

$$
\begin{aligned}
& \text { (a) } a_{0}+a_{1} z+\cdots+a_{k} z^{k} \neq 0 \\
& \text { (b) } a_{k+1}+a_{k+2} z+\cdots+a_{n} z^{n-k-1} \neq 0
\end{aligned}
$$

(2) $|z|=R$ implies

$$
\left|a_{0}+a_{1} z+\cdots+a_{k} z^{k}\right| \leq\left|a_{k+1} z^{k+1}+\cdots+a_{n} z^{n}\right|
$$

then $f(z)$ has at least $k+1$ zeros $z$ such that $0<|z| \leq R$.

There is an algebraic algorithm for determining the number of distinct zeros of a polynomial [4; page 65]. However, the algorithm offers no information on the norms of the zeros and, more significantly, since it considers one polynomial at a time it fails to identify classes of polynomials with a specific number of distinct zeros. On the other hand, Proposition 3 can be used to put conditions on the coefficients of the polynomial that imply the existence of many zeros, as follows.

COROLlary. If a polynomial

$$
f(z)=a_{0}+a_{1} z+\cdots+a_{n} z^{n}
$$

is such that, for some $k$ with $0<k<n$ and some $R>0$.

$$
\sum_{j=1}^{k}\left|a_{j}\right| R^{j}<\left|a_{0}\right|<2\left|a^{k+1}\right| R^{k+1}-\sum_{j=1}^{n}\left|a_{j}\right| R^{j}
$$

then $f(z)$ has at least $k+1$ distinct zeros $z$ such that $0<|z| \leq R$.

Proof. Hypothesis (1a) of Proposition 3 follows from the left-hand inequality and the triangle inequality because together they imply $\mid a_{0}+$ $a_{1} z+\cdots+a_{k} z^{k} \mid>0$. Since the right-hand inequality certainly implies

$$
\left|a_{k+1}\right| R^{k+1}>\left|a_{k+2}\right| R^{k+2}+\cdots+\left|a_{n}\right| R^{n},
$$


the same argument verifies hypothesis (1b). The full power of the righthand inequality, again with the aid of the triangle inequality, permits us to obtain hypothesis (2) of Proposition 3.

It is not difficult to find polynomials whose coefficients satisfy the hypotheses of the Corollary. A class of such examples is the following.

EXAMPLE. If $f(z)=a_{0}+a_{1} z+\cdots+a_{n} z^{n}$ is a polynomial whose coefficients satisfy the conditions

(i) $\left|a_{0}\right|>\left|a_{1}\right|+\cdots+\left|a_{k}\right|$

(ii) $\left|a_{k+1}\right|>\left|a_{0}\right|+\cdots+\left|a_{k}\right|+\left|a_{k+2}\right|+\cdots+\left|a_{n}\right|$ then $f(z)$ has at least $k+1$ distinct zeros such that $0<|z|<1$.

Proof. Letting

$$
R=\left(\left|2 a_{k+1}\right|^{-1} \sum_{j=0}^{n}\left|a_{j}\right|\right)^{1 / k+1}<1,
$$

and noting that

$$
\left|a_{0}\right|=2\left|a_{k+1}\right| R^{k+1}-\sum_{j=1}^{n}\left|a_{j}\right|
$$

it is easy to see that the inequalities of the Corollary hold.

The polynomial $f(z)=a_{0}+a_{n} z^{n}$ obviously has $n$ distinct zeros and, if $0<\left|a_{0}\right|<\left|a_{n}\right|$, then for each zero $z$ we know $0<|z|<1$. We would therefore expect that if $f(z)=a_{0}+a_{1} z+\cdots+a_{n} z^{n}$ where $\left|a_{n}\right|$ is larger than $\left|a_{0}\right|$, and both are much larger than $\left|a_{j}\right|$ for $0<j<n$, then $f(z)$ should still have $n$ distinct zeros $z$, all with $0<|z|<1$. The Example, for the case $k+1=n$, supplies some precision to this observation because it states that this conclusion holds whenever

$$
\left|a_{0}\right|>\left|a_{1}\right|+\cdots+\left|a_{n-1}\right|
$$

and

$$
\left|a_{n}\right|>\left|a_{0}\right|+\left|a_{1}\right|+\cdots+\left|a_{n-1}\right| .
$$

For a really specific example, consider

$$
f(z)=5+z+z^{2}+z^{3}+z^{4}+10 z^{5}
$$

then the Example tells us $f(z)$ has 5 distinct zeros $z$ and the formula for $R$ in its proof further tells us that for each zero we have $0<|z| \leq(.45)^{1 / 5}<$ .86 . 


\section{REFERENCES}

[1] R. Burckel, An Introduction to Classical Complex Analysis, Vol. 1, Academic Press, 1979.

[2] H. Hopf, Über Minimalzahl von Fixpunkten, Math. Z., 26 (1927), 762-774.

[3] B. Jiang, Lectures on Nielsen Fixed Point Theory, Contemporary Math., 14 (1983).

[4] J. Uspensky, Theory of Equations, McGraw-Hill, 1948.

Received October 3, 1983 and in revised form December 15, 1983.

UNIVERSITY OF CALIFORNIA, LOS ANGELES

LOS ANGELES, CA 90024 


\title{
PACIFIC JOURNAL OF MATHEMATICS EDITORS
}

DONALD BABBITT (Managing Editor)
University of California
Los Angeles, CA 90024
CHARLEs R. DEPrIMA
California Institute of Technology
Pasadena, CA 91125
R. FINN
Stanford University
Stanford, CA 94305

DoNAld BABBitT (Managing Editor)

Hermann FlaschKa

University of Arizona

Tucson, AZ 85721

RAmesh A. Gangolli

University of Washington

Seattle, WA 98195

ROBION KIRBY

University of California

Berkeley, CA 94720

C. C. MOORE

University of California

Berkeley, CA 94720

\author{
Hugo Rossi \\ University of Utah \\ Salt Lake City, UT 84112 \\ H. SAMELSON \\ Stanford University \\ Stanford, CA 94305 \\ HAROLD STARK \\ University of California, San Diego \\ La Jolla, CA 92093
}

ASSOCIATE EDITORS

R. ARENS

E. F. BECKENBACH
(1906-1982)

B. H. NEUMANN

F. WOLF

K. Yoshida

\section{SUPPORTING INSTITUTIONS}

UNIVERSITY OF ARIZONA

UNIVERSITY OF BRITISH COLUMBIA

CALIFORNIA INSTITUTE OF TECHNOLOGY

UNIVERSITY OF CALIFORNIA

MONTANA STATE UNIVERSITY

UNIVERSITY OF NEVADA, RENO

NEW MEXICO STATE UNIVERSITY

OREGON STATE UNIVERSITY
UNIVERSITY OF OREGON

UNIVERSITY OF SOUTHERN CALIFORNIA

STANFORD UNIVERSITY

UNIVERSITY OF HAWAII

UNIVERSITY OF TOKYO

UNIVERSITY OF UTAH

WASHINGTON STATE UNIVERSITY

UNIVERSITY OF WASHINGTON 


\section{Pacific Journal of Mathematics}

\section{Vol. 118, No. $1 \quad$ March, 1985}

Dan Amir, On Jung's constant and related constants in normed linear spaces ...1 Abdul Aziz, On the location of the zeros of certain composite polynomials . . 17 Joseph Barback, On hereditarily odd-even isols and a comparability of

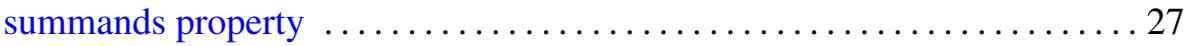

Matthew G. Brin, Klaus Johannson and Peter Scott, Totally peripheral 3-manifolds ........................................ 37

Robert F. Brown, A topological bound on the number of distinct zeros of an

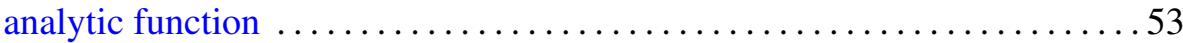

K. C. Chattopadhyay, Not every Lodato proximity is covered .......... 59

Beverly Diamond, Some properties of almost rimcompact spaces . .......63 63

Manfred Dugas and Rüdiger Göbel, On radicals and products . ......... 79

Abdelouahab El Kohen, A hyperbolic problem .................. 105

Harry Gonshor, Remarks on the Dedekind completion of a nonstandard model of the reals ................................... 117

William H. Kazez, On equivalences of branched coverings and their action

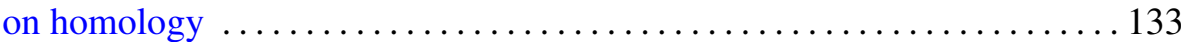

Darrell Conley Kent, On the Wallman order compactification .......... 159

Martin Andrew Magid, Lorentzian isoparametric hypersurfaces . . . . . . 165

Milan Miklavčič, Stability for semilinear parabolic equations with noninvertible linear operator

Richard Dean Neidinger and Haskell Paul Rosenthal, Norm-attainment of linear functionals on subspaces and characterizations of Tauberian operators

Johannes Vermeer, Closed subspaces of $H$-closed spaces 